Ciência Florestal, Santa Maria, v. 26, n. 1, p. 331-344, jan.-mar., 2016

ISSN 0103-9954

\title{
COMPOSIÇÃO FLORÍSTICA E CARACTERIZAÇÃO SUCESSIONAL COMO SUBSÍDIO PARA CONSERVAÇÃO E MANEJO DO PNMCBio, SOROCABA - SP
}

\author{
FLORISTIC AND SUCCESSIONAL CHARACTERIZATION AS A SUPPORT FOR CONSERVATION \\ AND MANAGEMENT OF PNMCBio, SOROCABA/SP
}

\author{
Samuel Coelho ${ }^{1}$ Eliana Cardoso-Leite ${ }^{2}$ Ana Carolina Devides Castello ${ }^{3}$
}

\begin{abstract}
RESUMO
O trabalho foi realizado no Parque Natural Municipal Corredores da Biodiversidade (PNMCBio), em Sorocaba -SP, com o objetivo de avaliar a composição florística do componente arbóreo e o estágio sucessional de uma área de floresta nativa de aproximadamente 31 hectares. Para tanto, foram alocadas 64 parcelas de tamanho $10 \times 10 \mathrm{~m}$, distribuídas aleatoriamente na área, sendo coletados todos os indivíduos com CAP igual ou maior que $15 \mathrm{~cm}$. Estimou-se a diversidade por meio do índice de diversidade de Shannon (H') e a equabilidade por meio do índice de Pielou (J'). As espécies foram caracterizadas nos grupos sucessionais, síndrome de dispersão, categorias de ameaça, amplitude de distribuição e indicadoras do estágio de regeneração. Foram amostrados 1088 indivíduos, distribuídos em 79 espécies, 54 gêneros e 29 famílias, sendo o H' estimado em 3,421 e o J' em 0,78, valores próximos aos encontrados em outras áreas de formações vegetacionais similares. Foram identificadas quatro espécies ameaçadas de extinção, 11 espécies endêmicas da Mata Atlântica e duas espécies restritas à Floresta Estacional Semidecidual no domínio da Mata Atlântica. O fragmento encontra-se em estágio médio de regeneração, sendo de suma importância sua conservação e manejo para a manutenção da biodiversidade local e regional.
\end{abstract}

Palavras-chave: Floresta Estacional Semidecidual; estágio sucessional; manejo.

\begin{abstract}
The study was conducted at the Natural Municipal Park "Corredores da Biodiversidade" (PNMCBio) in Sorocaba-SP, with the aim to evaluate the floristic composition of the tree component and the successional stage of a forest with about 31 hectares. Thereunto, were allocated 64 plots of 10 x 10 meters randomly distributed in the area. All individuals with $\mathrm{CBH}$ (circumference at the breast height) equal to or greater than $15 \mathrm{~cm}$ were included. We estimatedthe diversity through the Shannon diversity index ( $\left.\mathrm{H}^{\prime}\right)$ and evenness through the Pielou index ( $\left.\mathrm{J}^{\prime}\right)$. The species were characterized in successional groups, dispersal syndromes, categories of threat, distributional range and indicator of the regeneration stage. We sampled 1088 individuals distributed into 79 species, 54 genera and 29 families. The H' was estimated in 3,421 and the J' in 0.78 , values close to those found in other areas of similar vegetation formations. We sampled four endangered species, 11 Atlantic Forest endemic species and two species restricted to Seasonal Semideciduous Forest in the Atlantic Forest domain. The fragment is in an intermediate stage of succession, being of paramount importance to conservation and management for the maintenance of local and regional biodiversity.
\end{abstract}

Keywords: seasonal semideciduous forest; successional stage; management.

1 Biólogo, MSc., Laboratório de Ecologia, Universidade Federal de São Carlos, Campus de Sorocaba, Rod. João Leme dos Santos (SP-264), Km 110, At-Lab 117, Bairro do Itinga, CEP 18052-780, Sorocaba (SP), Brasil.samucabeca@hotmail.com

2 Bióloga, Dr ${ }^{\mathrm{a}}$., Professor Adjunto do Departamento de Ciências Ambientais, Laboratório de Ecologia, Universidade Federal de São Carlos, Campus de Sorocaba, Rod. João Leme dos Santos (SP-264), Km 110, At-Lab 117, Bairro do Itinga, CEP 18052-780, Sorocaba (SP), Brasil. cardosoleite@yahoo.com.br

3 Engenheira Florestal, Doutoranda do Programa de Pós-Graduação em Ciências Biológicas (Botânica), Universidade Estadual Paulista "Júlio de Mesquita Filho", Campus de Botucatu, Distrito de Rubião Júnior, s/n, CEP 18618-000, Botucatu (SP), Brasil. carol.dcastello@gmail.com

Recebido para publicação em 3/06/2013 e aceito em 3/04/2014

Ci. Fl., v. 26, n. 1, jan.-mar., 2016 


\section{INTRODUÇÃO}

Abrigando uma grande complexidade biológica, atualmente a Mata Atlântica é considerada um dos biomas mais ameaçados do mundo (DAVIS et al., 1986; MITTERMEIER et al., 1999), uma vez que restam aproximadamente $16 \%$ dos seus ecossistemas associados (RIBEIRO et al., 2009). Esta formação vegetacional configura-se em uma das áreas terrestres prioritárias para a conservação no globo (MYERS et al., 2000), com uma grande riqueza de espécies e diversidade florística e um grau de endemismo elevado para alguns grupos vegetais (DONADIO et al., 2009). Além da pequena área de Mata Atlântica e de grande parte ser de formações florestais secundárias, a maioria dos fragmentos tendem a ser pequenos, com mais de $80 \%$ deles possuindo menos de 50 hectares (RIBEIRO et al., 2009).

No interior do estado de São Paulo, além de sofrerem ameaças graves e constantes por estarem localizados próximos aos centros urbanos ou envolvidos por grandes monoculturas (FISZON, 2003; TABARELLI et al., 2005), são poucas as Unidades de Conservação que protegem tais formações (METZGER e RODRIGUES, 2008; XAVIER et al., 2008), principalmente as de Floresta Estacional (FES). A conservação desses ambientes é de fundamental importância, uma vez que a Mata Atlântica no estado é caracterizada por uma predominância de espécies com baixa frequência e com uma distribuição restrita (CAIAFA e MARTINS, 2010), sendo muitas delas ameaçadas de extinção. No estado de São Paulo e do Rio de Janeiro são poucos os estudos sobre a composição e estrutura da vegetação que têm identificado espécies ameaçadas (CARDOSO-LEITE et al., 2013).

A região de Sorocaba é a segunda maior em área de fragmentos florestais remanescentes do estado, perdendo apenas para a região do litoral (KRONKA et al., 2005) e se encontra intensamente urbanizada. Em Sorocaba, um desses fragmentos é parte da área do Parque Natural Municipal Corredores da Biodiversidade (PNMCBio), primeira Unidade de Conservação (UC) que está sendo efetivamente implantada no município. Devido às ações de implementação desta UC serem recentes, pouco se sabe sobre a vegetação nativa deste fragmento, o que dificulta o estabelecimento de ações que visem ao manejo; no entanto, sabe-se que o fragmento é bastante alterado (SOROCABA, 2012).
Os distúrbios ocasionados nos ecossistemas os tornam frágeis, aumentando a preocupação ambiental e destacando a necessidade de realizar estudos que subsidiem ações que mantenham a sustentabilidade dos fragmentos (GENELETTI, 2004). O crescente número de estudos realizados, analisando-se o grupo sucessional e a síndrome de dispersão das espécies, tem mostrado que estas características são fundamentais para resolver importantes questões ecológicas na escala dos ecossistemas, paisagens e biomas (WOODWARD e DIAMENT, 1991; LOPES et al., 2011), uma vez que incluem as respostas da vegetação a variações ambientais e regimes de perturbação (CORNELISSEN et al., 2003). A avaliação do estágio sucessional dos fragmentos é um subsídio importante para a conservação e recuperação das formações florestais, sendo a classificação das espécies em grupos ecológicos essencial para o entendimento do comportamento das espécies e da sucessão ecológica florestal (PAULA et al., 2004; LOPES et al., 2011).

Apesar de a região apresentar inúmeros fragmentos de floresta estacional (MELLO, 2012), são raros os estudos de composição florística e estrutura na região (ALBUQUERQUE e RODRIGUES, 2000; CARDOSO-LEITE e RODRIGUES, 2008). No caso do fragmento do PNMCBio, composto por Floresta Estacional Semidecidual (FES), conhecer as proporções de espécies e indivíduos nos grupos ecológicos se torna uma prioridade para as ações de manejo (LOPES et al., 2011), já que os modelos de recuperação de áreas degradadas devem seguir as distribuições dos grupos ecológicos mais comumente encontrados nos remanescentes conservados da região (PEREIRA et al., 2010). Frente à pluralidade de variantes ambientais, ressalta-se a importância de trabalhos descritivos que facilitem a compreensão da ecologia destas comunidades e direcionem futuros estudos (PRADO-JUNIOR et al., 2012). Assim, objetivouse incrementar as informações sobre a diversidade e distribuição das espécies vegetais que ocorrem na FES, apresentando a composição florística do fragmento remanescente do PNMCBio, bem como inferir sobre o seu estágio sucessional, fornecendo dados para embasar ações de manejo da área e da região. 


\section{MATERIAIS E MÉTODOS}

\section{Área do estudo}

A área do estudo faz parte do PNMCBio, localizado na zona norte do município de Sorocaba e entre as coordenadas $23^{\circ} 23^{\prime} 38^{\prime \prime} \mathrm{S} 47^{\circ} 29^{\prime} 04^{\prime \prime} \mathrm{O}$. A área total do Parque é de 62,42 ha, sendo que aproximadamente 31 ha refere-se à área de vegetação nativa do Parque, composto por Floresta Estacional Semidecidual e Floresta Estacional Semidecidual Aluvial (IBGE, 2012) (Figura 1). A porção sudoeste do fragmento, com menor altitude, configura-se como uma área de preservação permanente (APP), circundada pelo córrego Campininha, um afluente do rio Sorocaba. A altitude do fragmento varia de 570 a 620 metros, sendo que a formação pedológica presente é uma associação de Latossolos Vermelhos, caracterizados por serem distróficos a moderados, com textura argilosa e relevo suavemente ondulado e ondulado (IAC, 1999). Segundo a classificação Köppen (1948), o clima da região é uma transição de Cwb a Cwa, sendo tropical de altitude com verão moderadamente quente a quente e com temperatura média anual de aproximadamente $22^{\circ} \mathrm{C}$.

$\mathrm{O}$ fragmento estudado é circundado por plantios de Eucalyptus spp. manejados e abandonados, refletindo o alto grau de fragmentação de habitat da região, diretamente relacionado às atividades agropecuárias e ao processo de urbanização (ESTADO DE SÃO PAULO, 2012). O fragmento também sofre perturbações antrópicas, com visitas frequentes de moradores dos bairros próximos.

\section{Coleta e análise de dados}

A amostra foi de 64 parcelas (MUELLERDOMBOIS e ELLENBERG, 1974) de 10 m x 10 $\mathrm{m}$ alocadas aleatoriamente em diferentes porções do fragmento, com o objetivo de representar toda a vegetação. Foram amostrados todos os indivíduos com $15 \mathrm{~cm}$ ou mais de circunferência a altura do peito (CAP), incluindo os mortos que se encontravam em pé. As coletas botânicas foram realizadas durante o ano de 2012, sendo o material coletado, prensado e seco, conforme as técnicas usuais para este tipo de estudo (FIDALGO e BONONI, 1989). A identificação das espécies foi feita com base em literatura específica, por comparações com as coleções dos herbários CCTS e ESA (THIERS, 2013) e, quando necessário, auxílio de especialistas. Os nomes das famílias estão apresentados segundo o APG III (2009), sendo a grafia das espécies e nomes dos autores conferidos através da base de
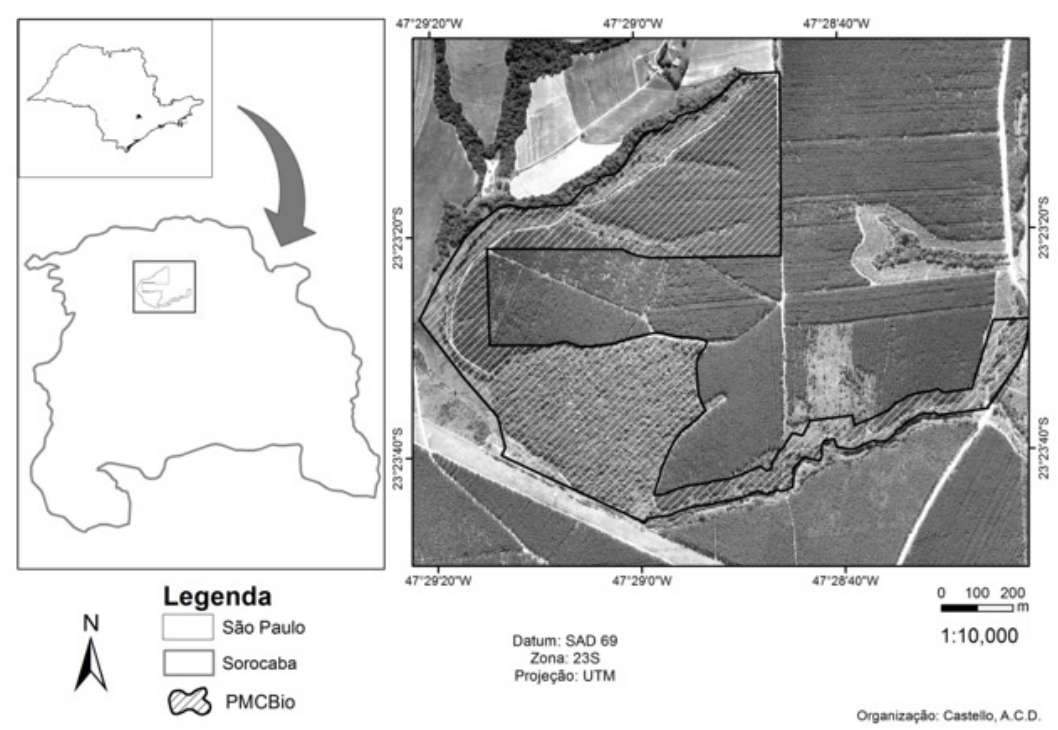

FIGURA 1: Localização do Parque Natural Municipal Corredores da Biodiversidade (PNMCBio) no município de Sorocaba - SP.

FIGURE 1: Natural Municipal Park Corredores da Biodiversidade (PNMCBio) location in Sorocaba/SP municipality. 
dados da Lista de Espécies da Flora do Brasil (2013) e do checklist das Spermatophyta do Estado de São Paulo, Brasil (WANDERLEY et al., 2011).

Para a estimativa da diversidade da área foi utilizado o índice de Shannon-Wiener (H'), com logaritmo na base natural, e o índice de diversidade de Simpson (D); para a equabilidade, que estima a igualdade florística entre os pontos amostrais, utilizou-se o índice de Pielou (J'), todos calculados através do software FITOPAC (SHEPHERD, 2009). A suficiência amostral foi testada por meio da curva do coletor (MUELLER-DOMBOIS e ELLENBERG, 1974). Considerou-se como espécies ameaçadas de extinção aquelas citadas como tal na Lista de Espécies da Flora Ameaçadas de Extinção do Estado de São Paulo (ESTADO DE SÃO PAULO, 2008), Lista de Espécies da Flora Ameaçadas de Extinção do Brasil (FUNDAÇÃO BIODIVERSITAS, 2008) e na Lista de Espécies da Flora Ameaçadas de Extinção da IUCN (IUCN, 2013). A lista da Fundação Biodiversitas foi utilizada para a análise em escala nacional por ser considerada mais abrangente do que a apresentada pelo Ministério do Meio Ambiente (BRASIL, 2008). Foram consideradas espécies endêmicas do Bioma Mata Atlântica, aquelas citadas por Stehmann et al. (2009) como endêmicas do Brasil, com distribuição geográfica restrita a este bioma.

A caracterização do grupo sucessional das espécies foi feita utilizando a compilação de dados da Secretaria do Estado do Meio Ambiente (ESTADO DE SÃO PAULO, 2008), considerandose duas grandes categorias: "Pioneiras" (P), que incluem as pioneiras e secundárias iniciais; e "Não Pioneiras" (NP), que agrupa as secundárias tardias e climáxicas, como também adotado por Viani (2005), Catharino et al. (2006) e Cardoso-Leite e Rodrigues (2008). Esta medida foi tomada para facilitar a interpretação dos dados e diminuir as chances de erros na identificação, uma vez que uma correta identificação dos grupos sucessionais dependeria de fatores como o conhecimento da biologia da espécie e da adaptação de outras publicações (CATHARINO et al., 2006). As espécies também foram identificadas quanto a sua síndrome de dispersão a partir da compilação de dados da Secretaria do Estado do Meio Ambiente (ESTADO DE SÃO PAULO, 2008), sendo incluídas em três categorias: zoocóricas, anemocóricas e autocóricas. Tanto para a classificação do grupo sucessional quanto para a síndrome de dispersão, espécies não identificadas até o epíteto específico e exóticas não foram caracterizadas (SC).

Para inferir sobre o estágio sucessional do fragmento foram considerados os seguintes parâmetros: a) proporção relativa entre o número de indivíduos e de espécies nos referidos grupos sucessionais (DISLICH et al., 2001; LIEBSCH et al. 2008); b) a proporção das síndromes de dispersão (LIEBSCH et al. 2008); e c) espécies indicadoras do estágio de regeneração (CONAMA, 1994). Para este último, calculou-se a proporção de indivíduos nos referidos grupos. O cálculo foi feito considerando o número total de indivíduos (excetuando-se os mortos), mesmo que nem todas as espécies tenham sido citadas pela Resolução.

\section{RESULTADOS E DISCUSSÃO}

Foram amostrados 1088 indivíduos, dos quais 116 encontravam-se mortos. Os indivíduos foram distribuídos em 29 famílias, 54 gêneros e 79 espécies (Tabela 1), somando-se mais uma que representa os indivíduos mortos. A curva do coletor estabilizou-se com 53 parcelas, havendo aumento apenas de uma espécie ao fim de toda amostra (64 parcelas), indicando que a amostragem foi suficiente para representar a composição florística do fragmento. Mesmo que não amostradas neste estudo, há outras espécies comuns nas formações vegetais de Sorocaba e são citadas por Koch et al. (2014), descrevendo qualitativamente a biodiversidade vegetal do município. No fragmento do PNMCBio, as famílias com maior riqueza foram Fabaceae (18), Myrtaceae (13), Euphorbiaceae (5), Lauraceae (4), Rutaceae (4), Salicaceae (4) e Sapindaceae (4), correspondendo a $65,8 \%$ das espécies amostradas. Destacam-se principalmente estas famílias por estar em conformidade com a grande riqueza de espécies encontrada em outros estudos realizados em regiões próximas à Sorocaba para formações de FES (IVANAUSKAS, 1999; ALBUQUERQUE e RODRIGUES, 2000; CARDOSO-LEITE et al., 2004; VIANI, 2005; BERNACCI et al., 2006; GUARATINI et al., 2008). Era esperado que essas famílias aparecessem entre as mais ricas em número de espécies, uma vez que estão entre as mais diversas do Brasil e são apontadas por vários autores, principalmente com relação às espécies lenhosas de grande porte. As famílias representadas com apenas uma espécie somaram 17 , excetuando-se as mortas, correspondendo a $60,7 \%$ das encontradas. Os gêneros com maior número de espécies foram Machaerium (7), Myrcia (7), Sebastiania (3), 
TABELA 1: Espécies amostradas no Parque Natural Municipal Corredores da Biodiversidade (PNMCBio), Sorocaba - SP. N.ind - Número de indivíduos coletados da respectiva espécie; GS - grupo sucessional ( $\mathrm{P}$ - pioneira, $\mathrm{NP}$ - não pioneira); $\mathrm{SD}$ - síndrome de dispersão (ZOO - zoocórica, ANE - anemocórica, AUT - autocórica); E - espécie indicadora de estágio de regeneração (EI - estágio inicial, EM - estágio médio, EA - estágio avançado); SC - sem caracterização; CA - categoria de ameaça; SP - Estado de São Paulo (ESTADO DE SÃO PAULO, 2008); IUCN - nível mundial (IUCN, 2013); QA - quase ameaçada; VU - vulnerável; ${ }^{1}$ espécie endêmica da Mata Atlântica.

TABLE 1: Species sampled at Natural Municipal Park "Corredores da Biodiversidade" (PNMCBio), Sorocaba/SP. N.ind - Number of specimens collected from the respective species; GS sucessional group ( $\mathrm{P}$ - pioneers, NP - non pioneers); SD - dispersion syndrome (ZOO zoochorous, ANE - anemochorous, AUT - autochorous); E - indicator species of regeneration stage (EI - initial stage, EM - medium stage, EA - advanced stage); SC - unclassified; CA - endangered status; SP - (state of) São Paulo (ESTADO DE SÃO PAULO, 2008); IUCN worldwide (IUCN, 2013); QA - near threatened; VU - vulnerable; ${ }^{1}$ Atlantic Forest endemic species.

\begin{tabular}{|c|c|c|c|c|c|c|}
\hline Família & Nome Científico & $\begin{array}{l}\mathrm{N} . \\
\text { Ind. }\end{array}$ & GS & SD & $\mathrm{CA}$ & $\mathrm{E}$ \\
\hline \multirow{2}{*}{ Anacardiaceae } & Lithrea molleoides (Vell.) Engl. & 3 & NP & $\mathrm{ZOO}$ & & \\
\hline & Tapirira guianensis Aubl. & 1 & $\mathrm{P}$ & $\mathrm{ZOO}$ & & EM \\
\hline Araliaceae & $\begin{array}{l}\text { Dendropanax cuneatus (DC.) Decne. \& } \\
\text { Planch. }\end{array}$ & 1 & NP & $\mathrm{ZOO}$ & & \\
\hline \multirow[b]{2}{*}{ Arecaceae } & Arecaceae sp & 4 & $\mathrm{SC}$ & $\mathrm{SC}$ & & \\
\hline & $\begin{array}{l}\text { Syagrus romanzoffiana (Cham.) } \\
\text { Glassman }\end{array}$ & 5 & $\mathrm{P}$ & $\mathrm{ZOO}$ & & \\
\hline Asteraceae & Gochnatia polymorpha (Less.) Cabrera & 3 & $\mathrm{P}$ & ANE & & EI \\
\hline Bignoniaceae & $\begin{array}{l}\text { Handroanthus umbellatus (Sond.) } \\
\text { Mattos }\end{array}$ & 13 & $\mathrm{P}$ & ANE & & EM \\
\hline Boraginaceae & $\begin{array}{l}\text { Cordia trichotoma (Vell.) Arráb. ex } \\
\text { Steud. }\end{array}$ & 11 & NP & ANE & & EM \\
\hline Burseraceae & Protium heptaphyllum (Aubl.) Marchand & 2 & $\mathrm{P}$ & $\mathrm{ZOO}$ & & \\
\hline Cannabaceae & Celtis fluminensis Carauta & 10 & $\mathrm{P}$ & $\mathrm{ZOO}$ & & \\
\hline Cardiopteridaceae & $\begin{array}{l}\text { Citronella paniculata (Mart.) R.A. } \\
\text { Howard }^{1}\end{array}$ & 7 & NP & $\mathrm{ZOO}$ & & \\
\hline Celastraceae & Maytenus gonoclada Mart. & 8 & $\mathrm{P}$ & $\mathrm{ZOO}$ & & \\
\hline Ebenaceae & Diospyros inconstans Jacq. & 11 & NP & $\mathrm{ZOO}$ & & \\
\hline Elaeocarpaceae & Sloanea monosperma Vell. & 2 & NP & $\mathrm{ZOO}$ & & \\
\hline \multirow{4}{*}{ Euphorbiaceae } & Croton floribundus Spreng. & 4 & $\mathrm{P}$ & AUT & & \\
\hline & Sebastiania brasiliensis Spreng. & 1 & $\mathrm{P}$ & AUT & & \\
\hline & $\begin{array}{l}\text { Sebastiania commersoniana (Baill.) L.B. } \\
\text { Sm. \& Downs }\end{array}$ & 1 & $\mathrm{P}$ & AUT & & \\
\hline & Sebastiania sp. & 1 & $\mathrm{SC}$ & $\mathrm{SC}$ & & \\
\hline
\end{tabular}


TABELA 1: Continuação...

TABLE 1: Continued...

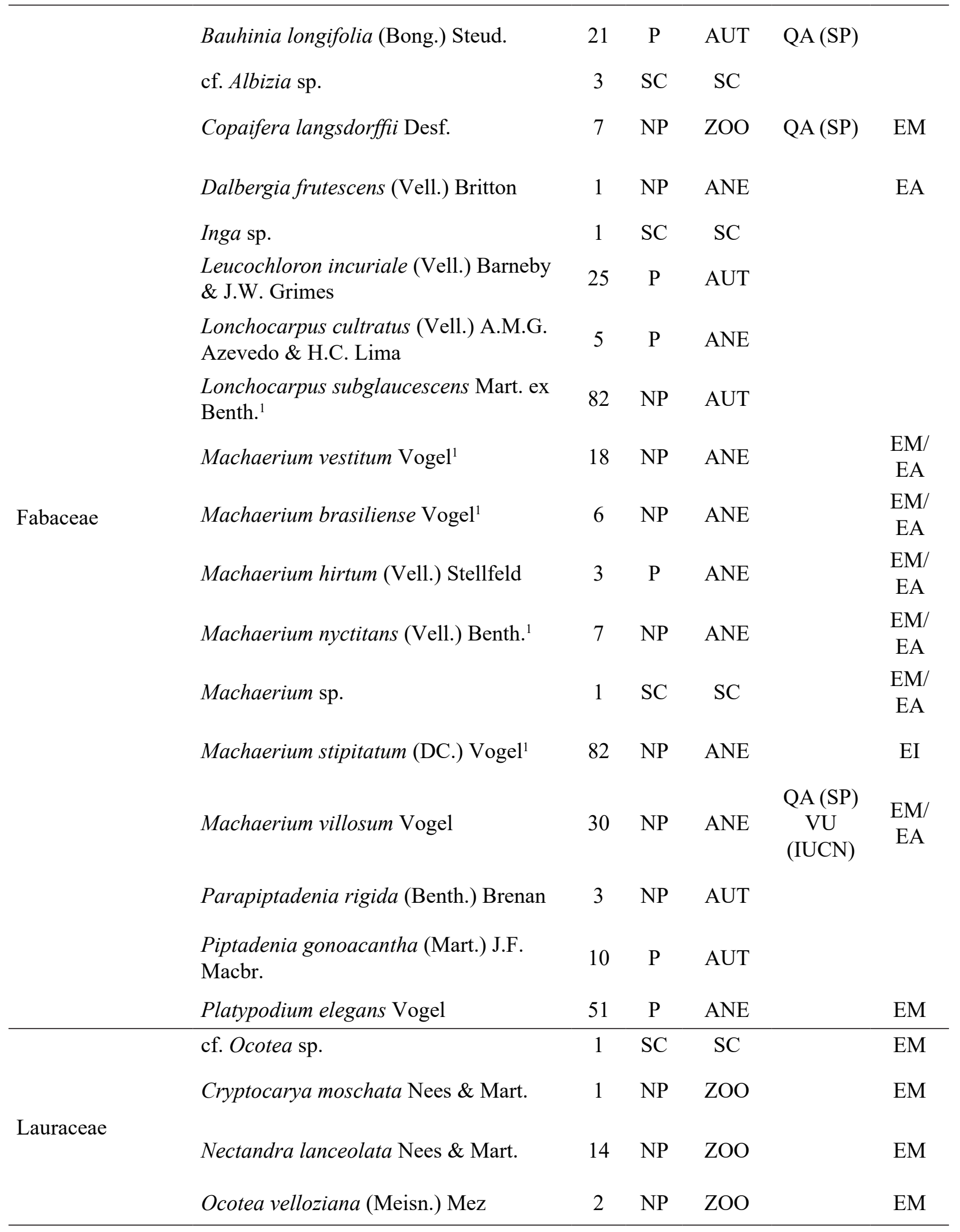

Continua...

Ci. Fl., v. 26, n. 1, jan.-mar., 2016 
TABELA 1: Continuação...

TABLE 1: Continued...

\begin{tabular}{|c|c|c|c|c|c|c|}
\hline Lecythidaceae & Cariniana estrellensis (Raddi) Kuntze & 1 & NP & ANE & $\mathrm{QA}(\mathrm{SP})$ & EA \\
\hline \multirow{3}{*}{ Malvaceae } & Guazuma ulmifolia Lam. & 1 & $\mathrm{P}$ & $\mathrm{ZOO}$ & & EI \\
\hline & Luehea divaricata Mart. & 7 & NP & ANE & & EM \\
\hline & Luehea grandiflora Mart. & 4 & NP & ANE & & EM \\
\hline Melastomataceae & Miconia minutiflora (Bonpl.) DC. & 1 & $\mathrm{P}$ & $\mathrm{ZOO}$ & & \\
\hline \multirow{3}{*}{ Meliaceae } & Cabralea canjerana (Vell.) Mart. & 1 & NP & $\mathrm{ZOO}$ & & EM \\
\hline & Trichilia elegans A. Juss. & 5 & NP & $\mathrm{ZOO}$ & & \\
\hline & Trichilia pallida $\mathrm{Sw}$. & 17 & $\mathrm{P}$ & $\mathrm{ZOO}$ & & \\
\hline Monimiaceae & Mollinedia widgrenii A. DC. & 2 & $\mathrm{NP}$ & $\mathrm{ZOO}$ & & \\
\hline \multirow[t]{6}{*}{ Moraceae } & Maclura tinctoria (L.) D. Don ex Steud. & 1 & NP & $\mathrm{ZOO}$ & & \\
\hline & $\begin{array}{l}\text { Campomanesia guazumifolia (Cambess.) } \\
\text { O. Berg }{ }^{1}\end{array}$ & 9 & NP & $\mathrm{ZOO}$ & & \\
\hline & Campomanesia sp & 2 & $\mathrm{SC}$ & $\mathrm{SC}$ & & \\
\hline & $\begin{array}{l}\text { Campomanesia xanthocarpa Mart. ex O. } \\
\text { Berg }^{1}\end{array}$ & 44 & NP & $\mathrm{ZOO}$ & & \\
\hline & Eugenia florida DC. & 1 & NP & $\mathrm{ZOO}$ & & \\
\hline & Eugenia paracatuana $\mathrm{O}$. Berg & 2 & $\mathrm{P}$ & $\mathrm{ZOO}$ & & \\
\hline \multirow[t]{8}{*}{ Myrtaceae } & Myrcia hebepetala DC. ${ }^{1}$ & 3 & NP & $\mathrm{ZOO}$ & & \\
\hline & Myrcia multiflora (Lam.) DC. & 3 & $\mathrm{P}$ & $\mathrm{ZOO}$ & & \\
\hline & Myrcia sp. 1 & 2 & $\mathrm{SC}$ & $\mathrm{SC}$ & & \\
\hline & Myrcia sp. 2 & 18 & $\mathrm{SC}$ & $\mathrm{SC}$ & & \\
\hline & Myrcia sp. 3 & 1 & $\mathrm{SC}$ & $\mathrm{SC}$ & & \\
\hline & Myrcia splendens (Sw.) DC. & 3 & $\mathrm{P}$ & $\mathrm{ZOO}$ & & \\
\hline & Myrcia tomentosa (Aubl.) DC. & 1 & NP & $\mathrm{ZOO}$ & & \\
\hline & $\begin{array}{l}\text { Myrciaria floribunda (H. West ex } \\
\text { Willd.) O. Berg }\end{array}$ & 1 & NP & $\mathrm{ZOO}$ & & \\
\hline Nyctaginaceae & Guapira opposita (Vell.) Reitz & 5 & NP & $\mathrm{ZOO}$ & & EI \\
\hline Peraceae & Pera glabrata (Schott) Poepp. ex Baill. ${ }^{1}$ & 26 & $\mathrm{P}$ & $\mathrm{ZOO}$ & & \\
\hline Primulaceae & $\begin{array}{l}\text { Myrsine coriacea (Sw.) R. Br. ex Roem. } \\
\text { \& Schult. }\end{array}$ & 1 & $\mathrm{P}$ & $\mathrm{ZOO}$ & & EI \\
\hline Rubiaceae & Cordiera concolor (Cham.) Kuntze & 2 & NP & $\mathrm{ZOO}$ & & \\
\hline
\end{tabular}


TABELA 1: Continuação...

TABLE 1: Continued...

\begin{tabular}{|c|c|c|c|c|c|}
\hline \multirow{4}{*}{ Rutaceae } & Citrus aurantium L. & 1 & $\mathrm{SC}$ & $\mathrm{SC}$ & \\
\hline & $\begin{array}{l}\text { Esenbeckia febrifuga (A. St.-Hil.) A. } \\
\text { Juss. ex Mart. }{ }^{1}\end{array}$ & 17 & NP & AUT & \\
\hline & Zanthoxylum rhoifolium Lam. & 9 & $\mathrm{P}$ & $\mathrm{ZOO}$ & EM \\
\hline & Zanthoxylum fagara (L.) Sarg. & 2 & NP & $\mathrm{ZOO}$ & EM \\
\hline \multirow{4}{*}{ Salicaceae } & Casearia obliqua Spreng. & 33 & NP & $\mathrm{ZOO}$ & \\
\hline & Casearia decandra Jacq. & 40 & $\mathrm{P}$ & $\mathrm{ZOO}$ & \\
\hline & Casearia sylvestris $\mathrm{Sw}$. & 93 & $\mathrm{P}$ & $\mathrm{ZOO}$ & EI \\
\hline & Xylosma pseudosalzmanii Sleumer & 2 & $\mathrm{P}$ & $\mathrm{ZOO}$ & \\
\hline \multirow{4}{*}{ Sapindacae } & Cupania vernalis Cambess. & 115 & $\mathrm{P}$ & $\mathrm{ZOO}$ & \\
\hline & $\begin{array}{l}\text { Allophylus edulis (A. St.-Hil., A. Juss. \& } \\
\text { Cambess.) Hieron. ex Niederl. }\end{array}$ & 5 & $\mathrm{P}$ & $\mathrm{ZOO}$ & \\
\hline & Diatenopteryx sorbifolia Radlk. & 1 & $\mathrm{P}$ & ANE & \\
\hline & Matayba elaeagnoides Radlk. & 28 & $\mathrm{P}$ & $\mathrm{ZOO}$ & EM \\
\hline Mortas & Mortas & 116 & $\mathrm{SC}$ & $\mathrm{SC}$ & \\
\hline
\end{tabular}

Campomanesia (3) e Casearia (3).

$\mathrm{O}$ índice de Shannon (H') foi estimado em 3,421 nats/ind. e o índice de equabilidade $\left(\mathrm{J}^{\prime}\right)$ foi de 0,78 , ambos os valores similares a outros estudos realizados em FES (IVANAUSKAS et al., 1999; IVANAUSKAS e RODRIGUES, 2000; DISLICH et al., 2001). Os valores de H' superiores a 3 nats/ind são comuns em estudos realizados em FES (DONADIO et al., 2009). O valor encontrado no fragmento indica que o mesmo possui uma diversidade relativamente elevada, uma vez que os trabalhos de Fonseca e Rodrigues (2000) e Donadio et al. (2009) apresentaram valores próximos a 2,7 nats/ind e consideraram as áreas com baixa diversidade. $\mathrm{O}$ valor encontrado para J' demonstra que a comunidade de espécies do fragmento possui uma distribuição similar de indivíduos por espécies (DONADIO et al., 2009). O valor do índice de diversidade de Simpson $(0,052)$ também aponta que a área possui uma diversidade relativamente alta, uma vez que quanto menor esse valor, menor a dominância e maior e diversidade. Este índice é complementar aos outros índices por considerar não só o número de espécies, mas também a uniformidade da distribuição da densidade (SCHAAF et al., 2006). Citrus aurantium foi a única espécie exótica amostrada, porém, também foi observada fora das parcelas, principalmente próximo à trilha do córrego Campininha. Acredita-se que a espécie tenha sido introduzida pela ação humana, uma vez que também foram encontrados resíduos (lixo) que indicam a prática de pesca. Essa espécie também foi encontrada no Parque Nacional do Iguaçu, sendo extremamente invasora, principalmente pelo seu longo período de frutificação e síndrome de dispersão zoocórica, por mamíferos e aves (RODOLFO et al., 2008). Desta forma, esta espécie merece atenção especial no controle e manejo, já que pode diminuir a disponibilidade da fauna para a dispersão de espécies nativas (RODOLFO et al., 2008) se sua densidade aumentar ao passar dos anos. As espécies nativas que apresentaram somente um indivíduo foram $21(26,58 \%)$, sendo esta uma proporção esperada para florestas tropicais (MARTINS, 1991; KLAUBERG et al., 2010). Mesmo que com baixa densidade, a presença destas espécies no fragmento é um forte indicativo da importância da conservação do mesmo, devendo priorizar essas espécies nos planos de restauração das áreas (SANTIN, 1999) e evitar a extinção local, caso esses indivíduos morram ou sofram corte (SILVA e SOARES, 2002).

Nenhuma espécie amostrada foi citada 
na lista da Fundação Biodiversitas (2008) como ameaçada; no entanto, foram encontradas quatro espécies ameaçadas, sendo três somente na lista do Estado de São Paulo (ESTADO DE SÃO PAULO, 2008) e uma na lista do Estado de São Paulo (ESTADO DE SÃO PAULO, 2008) e na lista da IUCN (IUCN, 2013) (Tabela 1). Machaerium villosum, que ocorre em Floresta Estacional dentro do bioma da Mata Atlântica (STEHMANN et al., 2009), foi citada tanto na lista estadual quanto na lista da IUCN, merecendo atenção nos esforços de conservação. Copaifera langsdorffii e Cariniana estrellensis apresentaram baixo número de indivíduos, demonstrando a importância das áreas protegidas e a necessidade de monitoramento para a conservação de suas populações.

Das 79 espécies amostradas neste estudo, 11 são endêmicas da Mata Atlântica (Tabela 1), sendo a família Fabaceae a que mais contribuiu neste valor, com cinco espécies. Na Mata Atlântica, o grau de endemismo é elevado (THOMAS et al., 1998; CAIAFA e MARTINS, 2010), sendo que determinadas espécies possuem uma distribuição geográfica restrita cujas populações são confinadas a habitat únicos (KRUCKEBERG e RABINOWITZ, 1985). Mesmo que não endêmicas do bioma Mata Atlântica, Machaerium villosum e Mollinedia widgrenii, quando presentes neste domínio, ocorrem somente em FES, demonstrando a importância da conservação de remanescentes deste ecossistema. Da mesma forma, apesar de o fragmento ser representado por FES, algumas espécies encontradas também são comuns a outras formações, como Cariniana estrellensis, Miconia minutiflora, Casearia obliqua, Casearia decandra e Xylosma pseudosalzmanii, espécies típicas de Floresta Ombrófila Densa (FOD) (STEHMANN et al., 2009).

Segundo a lista de São Paulo (2008), que também indica a ocorrência das espécies para o Cerrado, Pera glabrata, Leucochloron incuriale, Machaerium villosum, Platypodium elegans, Trichilia pallida e Esenbeckia febrifura, que apresentaram pelo menos 17 indivíduos na amostra, também ocorrem nesta formação e em FOD. Excetuando-se as espécies não identificadas até o epíteto específico, 37 são apontadas como ocorrentes em formações de Cerrado e, 53, em matas ciliares (ESTADO DE SÃO PAULO, 2008). Muitas espécies são comuns a outras tipologias florestais e, mesmo com baixa densidade, são de fundamental importância para a manutenção da comunidade vegetal e fauna associada. Desde décadas passadas, áreas próximas a Sorocaba receberam influências de outras formações vegetacionais, das Florestas Ombrófilas (Densa e Mista) ao Leste e ao Sul, e das formações savânicas a Oeste, constituindose em uma região fitogeograficamente ecotonal (ALBUQUERQUE e RODRIGUES, 2000).

A baixa densidade de algumas espécies pode ser justificada por motivos sucessionais ou serem espécies de outras formações (HACK et al., 2005). Estudos anteriores já relataram que Casearia decandra e Matayba elaeagnoides aumentam em densidade à medida que espécies pioneiras diminuem em vitalidade e abundância (KLEIN, 1960; KLAUBERG et al., 2010). Já Cupania vernalis, com sua autoecologia indicando ser heliófita ou de luz difusa, alcança estágios avançados na sucessão mais rapidamente que outras espécies (HACK et al., 2005).

Os indivíduos de espécies pioneiras - $\mathrm{P}$ contribuíram com mais da metade dos amostrados (Tabela 2), o que indica que o fragmento estudado se encontra em um estágio inicial de sucessão (DISLICH et al., 2001) se for considerado apenas este critério para sua classificação. Outros trabalhos, que também utilizaram esse critério para inferir sobre o estágio de sucessional, encontraram $65 \%$ e $75 \%$ de espécies P (DISLICH et al., 2001; CARDOSO-LEITE et al., 2004), o que indica que o fragmento do PNMCBio encontra-se em um estágio mais avançado quando comparado com as áreas de São Paulo e Rio Claro, estudadas por estes autores. Em relação às espécies, as não pioneiras NP contribuíram com 45,6\% (36), as P com 40,5\% (32), e 13,9\% ficaram sem categorização (Tabela 2). Os valores de espécies NP são inferiores aos encontrados para florestas maduras (TABARELLI e MANTOVANI, 1999; LIEBSCH et al., 2008), porém, deve-se considerar que os trechos estudados são de FES, formação que possui uma dinâmica própria, ou seja, há um período em que ocorre uma entrada maior de luz, devido à deciduidade de algumas espécies que a compõem (GANDOLFI, 2000), o que favorece o estabelecimento de espécies pioneiras. Além disso, o número de NP foi expressivo e, considerando também o fato de representarem boa proporção de indivíduos dentro do respectivo grupo, pode ser uma indicação de que o fragmento está assumindo estágio médio de regeneração.

A zoocoria representou a maior porcentagem de espécies e indivíduos amostrados, 
TABELA 2: Porcentagem (\%) de indivíduos e espécies amostradas na área do PNMCBio, município de Sorocaba - SP, nos distintos estágios sucessionais.

TABLE 2: Percentage (\%) of individuals and species sampled at the PNMCBio, Sorocaba municipality, SP, in different successional stages.

\begin{tabular}{lcc}
\hline \multicolumn{1}{c}{ Grupo sucessional } & $\begin{array}{c}\text { Indivíduos (número/ } \\
\text { porcentagem) }\end{array}$ & $\begin{array}{c}\text { Espécies (número/ } \\
\text { porcentagem) }\end{array}$ \\
\hline $\mathrm{P}$ (pioneiras + secundárias iniciais) & $510 / 52,5$ & $32 / 40,5$ \\
\hline $\mathrm{NP}$ (secundárias tardias + climácicas) & $427 / 43,9$ & $36 / 45,6$ \\
\hline SC (sem caracterização) & $35 / 3,6$ & $11 / 13,9$ \\
\hline
\end{tabular}

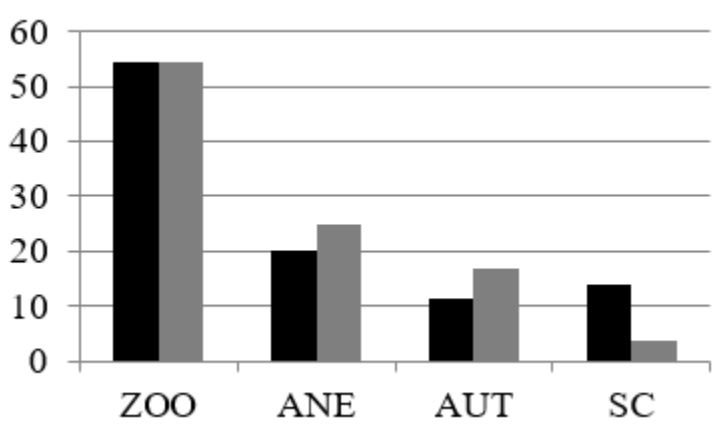

FIGURA 2: Porcentagem de espécies (preto) e indivíduos (cinza) nas síndromes de dispersão amostradas no PNMCBio em Sorocaba - SP. ZOO - Zoocórica; ANE - Anemocórica; AUT Autocórica; SC - Sem Caracterização.

FIGURE 2:Percentage of species (black) and individuals (gray) in the dispersion syndrome sampled at PNMCBio in Sorocaba/SP. ZOO - Zoochorous; ANE - Anemochorous; AUT Autochorous; SC - Unclassified.

$54,4 \%$ e $54,5 \%$ respectivamente, seguida por anemocoria, com $20,3 \%$ das espécies e $25 \%$ dos indivíduos, e autocoria, com $11,4 \%$ das espécies e $16,9 \%$ dos indivíduos, restando $13,9 \%$ das espécies e 3,6\% dos indivíduos não caracterizados (Figura 2). A predominância da síndrome zoocórica segue os padrões de florestas tropicais (HOWE e SMALLWOOD, 1982), principalmente nas estacionais (KINOSHITA et al., 2006; RUSCHEL et al., 2007). Além disso, a maior proporção de espécies zoocóricas pode indicar que o fragmento possui características de estágios intermediários de sucessão (LIEBSCH et al., 2008; CARDOSO-
LEITE et al., 2013).

Em relação às espécies indicadoras do estágio de regeneração (CONAMA, 1994), seis espécies amostradas são consideradas indicadoras de estágio inicial, 15 são consideradas indicadoras de estágio médio, seis são consideradas indicadoras tanto de estágio médio quanto de estágio avançado e duas espécies são consideradas indicadoras de estágios avançados. Quanto ao número de espécies e indivíduos, houve uma maior proporção daquelas indicadoras de estágio médio e/ou avançado de regeneração ( $22,8 \%$ dos indivíduos), contrastando com a menor proporção de espécies e indivíduos indicadores de estágio inicial (19\% dos indivíduos). Tais valores, aliados aos demais resultados, são mais um indicativo de que o fragmento encontra-se em estágio médio de regeneração.

\section{CONCLUSÃO}

Apesar de se tratar de um fragmento de FES relativamente pequeno que está evoluindo ao estágio médio de regeneração, este apresentou um $\mathrm{H}^{\prime}$ relativamente alto e comparável a outras áreas de FES e uma proporção de espécies zoocóricas também alta, o que contribui para a conservação da fauna associada a esta formação. Além disso, o mesmo abriga espécies endêmicas e ameaçadas, o que justifica sua importância para conservação e manutenção da biodiversidade local e regional, cumprindo com um dos objetivos da criação do PNMCBio. Em futuras ações de recuperação de áreas degradadas da região, a possibilidade de conexão do remanescente com outros fragmentos é um fator a ser considerado, uma vez que um grande corredor biológico pode ser formado associado a áreas de preservação permanente nesta região do município. 


\section{AGRADECIMENTOS}

À Toyota do Brasil pelo auxílio financeiro, aos amigos pelo auxílio no trabalho de campo, à especialista Fiorella Fernanda Mazine Capelo (Myrtaceae - UFSCar-Sorocaba) e ao generalista Marcelo A. Pinho Ferreira (ESALQ/USP) pela ajuda na identificação do material botânico.

\section{REFERÊNCIAS BIBLIOGRÁFICAS}

ALBUQUERQUE, G.B.; RODRIGUES, R.R. A vegetação do Morro de Araçoiaba, Floresta Nacional de Ipanema, Iperó (SP). Scientia Forestalis, n. 28, p. 145-159, 2000.

APG. An update of the Angiosperm Phylogeny Group classification for the orders and families of flowering plants: APG III. Botanical Journal of the Linnean Society, v. 161, n. 2, p. 105- 202, 2009. BERNACCI, L.C. et al. O efeito da fragmentação florestal na composição e riqueza de árvores na região da Reserva do Morro Grande (Planalto de Ibiúna-SP). Revista do Instituto Florestal, São Paulo, v. 18, n. único, p. 121-166, 2006.

BRASIL. Ministério do Meio Ambiente. instrução normativa n.6, de 23 de Setembro de 2008. Lista Oficial das Espécies da Flora Brasileira Ameaçadas de Extinção. Disponível em: < http://www.mma.gov. br/estruturas/179/_arquivos/179_05122008033615. pdf $>$. Acesso em: 22 de maio de 2013.

CAIAFA, A.N.; MARTINS, F.R. Forms of rarity of tree species in the southern Brazilian Atlantic Rainforest. Biodiversity and Conservation, v. 19, p. 2597-2618, 2010.

CARDOSO-LEITE, E. et al. Fitossociologia e caracterização sucessional de um fragmento de mata ciliar, em Rio Claro/SP, como subsídio à recuperação da área. Revista do Instituto Florestal, São Paulo, v.16, n.1, p.31-41, 2004.

CARDOSO-LEITE, E.; RODRIGUES, R.R. Fitossociologia e caracterização sucessional de um fragmento de floresta estacional no sudeste do Brasil. Revista Árvore, Viçosa, v. 32, n. 3, p. 583595, 2008.

CARDOSO-LEITE, E. et al. Analysis of floristic composition and structure as an aid to monitoring protected areas of dense rain forest in southeastern Brazil. Acta Botanica Brasilica, v. 27, n. 1, p. 180-194, 2013.

CATHARINO, E.L.M. et al. Aspectos da composição e diversidade do componente arbóreo das florestas da Reserva Florestal do Morro Grande, Cotia, SP. Biota Neotropica, v. 6, n. 2, p. 1-18, 2006.

CONAMA. Resolução no. 1 , de 31 de janeiro de 1994. Regulamenta o Decreto no. 750 , de 10 de fevereiro de 1993 e a Resolução CONAMA no. 10 de $1^{\circ}$ de outubro de 1993 em relação a necessidade de se definir vegetação primária e secundária nos estágios pioneiro, inicial, médio e avançado de regeneração de Mata Atlântica a fim de orientar os procedimentos de licenciamento de exploração nativa do Estado de São Paulo. Disponível em: <www.conama.gov.br>. Acesso em: 23 maio 2013. CORNELISSEN, J.H.C. et al. A handbook of protocols for standardized and easy measurement of plant functional traits worldwide. Australian Journal of Botany [online], v. 51, p. 335-380, 2003.

DAVIS, S.D. et al. Plants in danger: what do we know?. Switzerland and Cambridge: Gland-IUCN, 1986. 444p.

DISLICH, R.; CERSÓSIMO, L.; MANTOVANI, W. Análise da estrutura de fragmentos florestais no Planalto Paulistano-SP. Revista Brasileira de Botânica, v.24, n.3, p.321-332, 2001.

DONADIO, N.M.M.; PAULA, R.C. de.; GALBIATTI, J.A. Florística e estrutura da comunidade arbórea de um remanescente florestal ripário no município de Guariba, Estado de São Paulo, Brasil. Revista do Instituto Florestal, São Paulo, v. 21, n. 1, p. 1-17, 2009.

ESTADO DE SÃO PAULO. Secretaria de Estado do Meio Ambiente. Resolução SMA n ${ }^{\circ} 08$ de 31 de janeiro de 2008. Fixa a orientação para o reflorestamento heterogêneo de áreas degradadas e dá providências correlatas, 2008.

ESTADO DE SÃO PAULO. Secretaria do Meio Ambiente / Coordenadoria de Planejamento Ambiental. Meio Ambiente Paulista: Relatório de Qualidade Ambiental 2012. Organização: Fabiano Eduardo Lagazzi Figueiredo. São Paulo: SMA/ CPLA, 2012.

FIDALGO, O.; BONONI, V.L.R. Técnicas de coleta, preservação e herborização de material botânico. São Paulo: Instituto de Botânica/Imprensa Oficial do Estado de São Paulo, 1989, 62p.

FISZON, J.T. et al. Causas Antrópicas - 03. In: RAMBALDI, D. M.; OLIVEIRA, D. A. S. de (Orgs.). Fragmentação de Ecossistemas: Causas, efeitos sobre a biodiversidade e recomendações de políticas públicas Brasília: MMA/SBF, 2003. p. 65- 99 .

FONSECA, R.C.B.; RODRIGUES, R.R. Análise 
estrutural e aspectos do mosaico sucessional de uma floresta semidecídua em Botucatu,SP. Scientia Forestalis, Piracicaba, n. 57, p. 27-43, 2000.

FUNDAÇÃO BIODIVERSITAS. Lista oficial de espécies ameaçadas de extinção no Brasil. 2008. Disponível em: <http://www.biodiversitas.org.br/ florabr/consulta_fim.asp $>$. Acesso em: 22 de maio de 2013.

GANDOLFI, S. et al. Levantamento florístico e caráter sucessional das espécies arbustivo-arbóreas de uma floresta mesófila semidecídua no município de Guarulhos, SP. Revista Brasileira de Biologia, v. 55 , n. 4, p. 753-767, 1995.

GENELETTI, D. Using spatial indicators and value functions to assess ecosystem fragmentation caused by linear infrastructures. International Journal of Applied Earth Observation and Geoinformation, v. 5, n. 1, p.1-15. 2004.

GUARATINI, M.T.G. et al. Composição florística da Reserva Municipal de Santa Genebra, Campinas, SP. Revista Brasileira de Botânica, v. 31, n. 2, p. 323-337, 2008.

HACK, C. et al. Análise fitossociológica de um fragmento de floresta estacional decidual no município de Jaguari, RS. Ciência Rural, Santa Maria, v. 35, n. 5, p. 1083-1091. 2005.

HOWE, H.; SMALLWOOD, J. Ecology of seed dispersal. Annual Review of Ecology and Systematics, v. 13, n. 1, p. 201-228, 1982.

IAC - INSTITUTO AGRONÔMICO DE CAMPINAS. Mapa Pedológico do Estado de São Paulo (relatório e mapa). 1: 500.000. Campinas: IAC, 1999.

IBGE - INSTITUO BRASILEIRO DE GEOGRAFIA E ESTATÍSTICA. Manual técnico da vegetação brasileira. Rio de Janeiro, 2012, 271p. (Manuais Técnicos em Geociências, n. 1).

IUCN - INTERNATIONAL UNION FOR CONSERVATION OF NATURE. Lista Vermelha de Espécies Ameaçadas de Extinção da União Internacional para a Conservação da Natureza. 2013. Disponível em: <http://www. iucnredlist. org >. Acesso em: 21 de maio de 2013.

IVANAUSKAS, N.M; RODRIGUES, R.R.; NAVES, A.G. Fitossociologia de um trecho de Floresta Estacional Semidecídua em Itatinga, SP, Brasil. Scientia Forestalis, Piracicaba, v. 56, p. 83-99, 1999.

IVANUSKAS, N.M.; RODRIGUES, R.R. Florística e fitossociologia de floresta estacional decidual em Piracicaba, São Paulo, Brasil. Revista Brasileira de Botânica, v. 23, n. 3, p. 291-304, 2000.

KINOSHITA, L.S. et al. Composição florística e síndromes de polinização e de dispersão da mata do Sítio São Francisco, Campinas, SP, Brasil. Acta Botanica Brasilica, v. 20, n. 2, p. 313-327, 2006.

KLAUBERG, C. et al. Florística e estrutura de um fragmento de Floresta Ombrófila Mista no Planalto Catarinense. Revista Biotemas v. 23, n. 1, p. 35-47, 2010.

KLEIN, R.M. O aspecto dinâmico do pinheiro brasileiro. Sellowia, v. 12, p. 17-45, 1960.

$\mathrm{KOCH}$, I. et al. Lista das plantas com flores e frutos das áreas de vegetação remanescente do município de Sorocaba. Pp. 85-151 In: Smith, W.S.; Mota Junior, V.D.; Carvalho, J.L. (org.) Biodiversidade do Município de Sorocaba. 2014.

KÖEPPEN, W. Climatologia: con un estudio de los climas de la tierra. México: Fundo de Cultura Econômica, 1948.

KRONKA, F.J.N. et al. Inventário florestal da vegetação natural do Estado de São Paulo. São Paulo: Imprensa Oficial, 2005. 200p.

KRUCKEBERG, A.R.; RABINOWITZ, D. Biological aspects of endemism in higher plants. Annual Review of Ecology and Systematic, v. 16, p. 447-479, 1985.

LIEBSCH, D.; MARQUES, M.C.M.; GOLDENBERG R. How long does the Atlantic Rain Forest take to recover after a disturbance? Changes in species composition and ecological features during secondary succession. Biological Conservation, v. 141, p. 1717-1725, 2008.

LOPES, S.F. et al. Caracterização ecológica e distribuição diamétrica da vegetação arbórea em um remanescente de Floresta Estacional Semidecidual na Fazenda Experimental do Glória, Uberlândia, MG. Bioscience Journal, v. 27, n. 2, p. 322-335, 2011.

MARTINS, F.R. Estrutura de uma floresta mesófila. Campinas: Editora da Unicamp. 1991. 246p.

MELLO, K. Análise espacial de remanescentes florestais como subsídio para o estabelecimento de unidades de conservação. 2012. 82f. Dissertação. (Mestrado em Diversidade Biológica e Conservação) - Universidade Federal de São Carlos, Centro de Ciências e Tecnologia para a Sustentabilidade, Sorocaba, São Paulo, 2012.

METZGER, J.P.; RODRIGUES, R.R. Mapassíntese das diretrizes para conservação e restauração da biodiversidade no Estado de São Paulo. In: 
SECRETARIA DO MEIO AMBIENTE. Diretrizes para conservação e restauração da biodiversidade no Estado de São Paulo. São Paulo: Secretaria de Estado do Meio Ambiente, 2008.

MITTERMEIER, R.A. et al. Hotspots: earth's biologically richest and most endangered terrestrial ecoregions. Mexico City: CEMEX/ Conservation International, 1999. 431p.

MUELLER-DOMBOIS, D.; ELLENBERG, H. Aims and methods of vegetation ecology. New York: Wiley \& Sons. 1974. 574p.

MYERS, N. et al. Biodiversity hotspots for conservation priorities. Nature, v.403, p.853-858, 2000.

PAULA, A. et al. Sucessão ecológica da vegetação arbórea em uma Floresta Estacional Semidecidual, Viçosa, MG, Brasil. Acta Botanica Brasilica, v. 18, n. 3, p. 407-423, 2004.

PEREIRA, I.M. et al. Caracterização ecológica de espécies arbóreas ocorrentes em ambientes de mata ciliar, como subsídio à recomposição de áreas alteradas nas cabeceiras do Rio Grande, Minas Gerais, Brasil. Ciência Florestal, Santa Maria, v. 20, n. 2, p. $235-253,2010$

PRADO-JÚNIOR, J.A. et al. Estrutura da comunidade arbórea em um fragmento de Floresta Estacional Semidecidual localizada na Reserva Legal da Fazenda Irara, Uberlândia, MG. Bioscience Journal, v. 26, n. 4, p. 638-647, 2010.

RIBEIRO, M.C. et al. The Brazilian Atlantic Forest: How much is left, and how is the remaining forest distributed? Implications for conservation. Biological Conservation, v. 142, p. 1141-1153, 2009.

RUSCHEL, A.R.; NODARI, R.O; MOERSCHBACHER, B.M. Woody plant species richness in the Turvo State Park, a large remnant of Deciduous Atlantic Forest, Brazil. Biodiversity and Conservation, v. 16, p. 1699-1714, 2007.

RODOLFO, A.M. et al. Citrus aurantium L. (laranja-apepu) e Hovenia dulcis Thunb. (uva-dojapão): espécies exóticas invasoras da trilha do Poço Preto no Parque Nacional do Iguaçu, Paraná, Brasil. Revista Brasileira de Biociências, v. 6, n. 1, p. 16-18, 2008.

SANTIN, D.A. A vegetação remanescente do município de Campinas (SP): mapeamento, caracterização fisionômica e florística, visando à conservação. 1999. 502f. Tese (Doutorado em Ciências Biológicas) -Universidade Estadual de Campinas, Campinas, 1999.
SCHAAF, L.B. et al. Modificações florísticoestruturais de um remanescente de floresta Ombrófila Mista Montana no período entre 1979 e 2000. Ciência Florestal, Santa Maria, v. 16, n. 3, p. 271-291, 2006.

SHEPHERD, G.J. FITOPAC 2.1. Universidade Estadual de Campinas. Depto de Botânica, 2009.

SILVA, L.A.; SOARES, J.J. Levantamento fitossociológico em um fragmento de floresta estacional semidecídua, no município de São Carlos, SP. Acta Botanica Brasilica, v. 16, n. 2, p. 205-216, 2002.

SOROCABA. Secretaria do Meio Ambiente. Plano de Manejo do Parque Natural Municipal Corredores da Biodiversidade de Sorocaba (versão preliminar). Biométrica. Sorocaba. 2012. $387 \mathrm{p}$.

STEHMANN, J.R. et al. Plantas da Floresta Atlântica. Rio de Janeiro: Jardim Botânico do Rio de Janeiro, 2009, 516p.

TABARELLI, M.; et al. Desafios e oportunidades para a conservação da biodiversidade da Mata Atlântica brasileira. Megadiversidade, v. 1, n. 1, 2005.

TABARELLI, M.; MANTOVANI, W. Clareiras naturais e riqueza de espécies pioneiras em uma Floresta Atlântica Montana. Revista Brasileira Biologia, v. 59, n. 2, p. 251-261, 1999.

THIERS, B. [continuously updated]. Index Herbariorum: A global directory of public herbaria and associated staff. New York Botanical Garden's Virtual Herbarium. Disponível em: <http:// sweetgum.nybg.org/ih/>. Acesso em: 07 maio 2013. THOMAS, W.W. et al. Plant endemism in two forests in southern Bahia, Brazil. Biodiversity and Conservation v. 7, p. 311-322, 1998.

VIANI, R.A.G. O uso da regeneração natural (floresta estacional semidecidual e talhões de Eucalyptus) como estratégia de produção de mudas e resgate da diversidade vegetal na restauração florestal. 2005. 118f. Dissertação (Mestrado em Biologia Vegetal). Universidade Estadual de Campinas, Campinas, 2005.

WANDERLEY, M.G. Checklist das Spermatophyta do Estado de São Paulo, Brasil. Biota Neotropica, v. 11, p. 193-390, 2011.

WOODWARD, F.I.; DIAMENT, A.D. Functional approaches to predicting the ecological effects of global change. Functional Ecology, v. 5, 
p. 202-212, 1991.

XAVIER, A.F.; BOLZANI, B.M.; JORDÃO, S. Unidades de Conservação da Natureza no Estado de São Paulo. In: In: SECRETARIA DO MEIO
AMBIENTE. Diretrizes para conservação e restauração da biodiversidade no Estado de São Paulo. São Paulo: Secretaria de Estado do Meio Ambiente, 2008. 